\title{
Mujeres rurales y sobrevivencia familiar
}

\author{
Aida Carolina Quinteros
}

\section{Introduccion}

En esle trabajo, a través de la problemática particular de la mujeres campesinas salvadorefias, queremos mostrar como el palriarcado y sus mitos sobre la siluación de la mujer no es un problema "de las mujeres", sino que es más bien un problema de toda la sociedad. La discriminación no sólo liene efeclos en la vida privada de las personas sino que puede, en algunos momenlos, ser un elemento más que contribuya al mantenimiento de una situación negativa para las familias. En tal sentido la llamada "perspectiva de género" no contribuye solamente a develar problemas de incumbencia para las mujeres, sino que además es un elemento útil para quienes se interesan por conocer el funcionamiento de las sociedades actuales y para quienes trabajan por mejorar las condiciones de vida de las poblaciones pobres.

Para la redacción de las siguienles páginas se ha tomado como base el trabajo de graduación presentado en julio de 1992 para optar al grado de licenciada en sociología de esta Universidad, titulado: "El papel de la mujer rural en la planificación para el desarrollo (un estudio de caso)"'. Dicho trabajo de graduación contó con la realización de varias entrevislas en prolundidad con mujeres rurales de la zona de Armenia, Sonso-

1. Quinteros A.C y Benltez G. "El papel de la mujer rural en la planificación para el desarrollo" (estudio de caso). Tesis para optar por la Licenciatura en Sociología de la Universidad Centroamericana "José Simeón Cañas" San Salvador julio 1992. 
nate. Muchos de los datos fueron tomados de eslas entrevistas, asi como de algunos talleres de rellexión realizados con mujeres de la misma zona.

\section{Los mitos del palrlarcado.}

Las sociedades patriarcales mantienen muchos mitos acerca de las condiciones en que en ella se desenvuelven las mujeres y los hombres. Sin embargo, estos mitos han resultado ser contradictorios con la realidad, en la mayorla de las veces. Trislemente, develar la realidad tras estos mitos no resulla tarea tácil y Irecuentemente muchos, inclusive aquellos que lienen en sus manos el desarrollo de proyectos o actividades tendientes al mejoramienlo de las comunidades pobres, caen en la trampa de considerar los supuestos patriarcales como ciertos, acarreando negativos resultados para las y los beneficiarios de tales proyeclos de desarrollo.

Uno de los errores más comunes es que se tiende a pensar que las mujeres campesinas trabajan solo en lo doméstico, siendo el hombre el encargado y responsable de realizar las tareas produclivas y de traer el sustenlo a la familia. En cuanto a este punto, son varias las investigaciones que han demostrado que las mujeres realizan tareas productivas más de lo que suele pensarse.

Oro de los mitos es el que liende a idenlíficar aulomáticamente al hombre como el jefe de la lamilia y por to tanto, como el beneliciario directo de políticas lales como la Reforma Agraria, olorgamientos de tierras, elc. Siendo que en la realidad, la mayoría de hogares están siendo jefeados por mujeres solas (divorciadas, abandonadas, viudas), 0 por mujeres que aunque estén acompafias, el hombre de hecho no mantiene una presencia constante en ese hogar.

Ciertamente detectar la jefalura de la familia no es un procedimiento tan sencillo como parece a simple vista, ya que los patrones culturales lienden a identificar a la ligura masculina como la cabeza de la tamilia per se y aunque se sabe que procesos lales como las migraciones temporales, irresponsabilidad patema y otros influyen para que en muchos casos la figura masculina del padre no tenga presencia en la vida cotidiana lamiliar, las mujeres tienden a declarar como jefe al hombre ausenle.

La situación de las mujeres jelas de tamilia es bastanle problemática para la sobrevivencia de ese grupo familiar ya que en primer lugar se trata de mujeres solas al Irente del hogar. Las mujeres que se declaran jelas de familia en las encueslas de hogares no declaran cónyuge en el 
95\% de los casos según Garcla y Gomáriz, 1989². En el caso de los hombres jeles la situación es diferenle ya que la mayoria declara conyuge, to cual significa que en este último caso, existen dos responsables de la manutención tamiliar y por tanto dos ingresos al hogar. Además probablemenle a través del hombre las familias logren hacerse de recursos que no los lograrian si son las mujeres las que estén al frente de la casa.

Ambos mitos tienen consecuencias nelaslas para las mujeres y para las lamilias rurales en general, ya que a pesar que las mujeres si Irabajan en tareas que generan productos e ingresos para las familias, y que son además jefas de la misma (declaradas o de hecho) en muchos casos, éstas no tienen acceso recursos productivos con los cuales hacerle Irente a la manutención de su familia. Los hombres campesinos también tienen dificultades, sin embargo las mujeres tienen posibilidades aun menores que los hombres para acceder a la tierra, a la cooperativa, a la asislencia técnica y a salarios suficientes.

Eslos mitos se consideran como verdaderos dentro de las concepciones de la sociedad y muy pocas veces son cueslionados por los estudiosos del campesinado y por los encargados de administrar políticas dirigidas al campo. Empero, su creencia redunda en delrimento de las familias campesinas.

\section{Las mujeres en la economia famillar.}

Comúnmente tiende a pensarse que las mujeres se dedican la mayor parte de su tiempo a tareas no productivas o al trabajo doméstico. Esta idea tiene su base en el hecho de que ciertamenle, la mujer es la principal encargada, la responsable casi única del trabajo de reproducción Iamiliar ${ }^{3}$.

Sin embargo, las mujeres no realizan solamente Irabajo reproduclivo,

2. todas las referencias bibliográlicas que se hacen en este artículo están tomadas tal y como aparecen en el trabajo de graduación ya citado.

3. El concepto de trabajo reproductivo se reliere a las actividades que se realizan en torno a la procreación y crianza de los hijos, reposición cotidiana de los miembros de la familia (alimentación, lavado de ropa, elc), cuido de los ancianos y entermos, transmisión de la cultura en el ámbito privado, cuido y limpieza de la casa, etc. estas tareas son realizadas en forma exclusiva bajo la responsabilidad de las mujeres. La división genérica del trabajo le asigna, de hecho, esta rama de la actividad social, como una tarea del género femenino. 
sino que desde la casa misma responden con trabajo productivo a las exigencias de sobrevivencia de su familia y participa activamente en su manutención.

Algunas datos que comprueban la atirmación anterior los proporciona una encuesta nacional de 2,152 hogares rurales realizada en República Dominicana. Según esa encuesta la participación laboral de las mujeres en el sistema agricola familiar se elevaba al $84 \%$, en contraste con el censo que registró solamente el $21 \%$ de las mujeres rurales como económicamente activas (CIPAF, 1985: 162). Por otro lado, otra investigación realizada en la región peruana indica que las tasas de participación llegaban a niveles del $86 \%$ y en "... la encuesta de hogares realizada por ACEP en la región de García Rovira en Colombia, se encontró que solamente en un $18 \%$ de los hogares la mujer participaba en el trabajo directo en el campo, pero el $24 \%$ lo hacía en el mercadeo, el $53 \%$ el procesamiento de los productos agricolas, el $88 \%$ en el cuidado de los animales y el $95 \%$ en las labores de cocina para mano de obra extrafamiliar (Deere y León, 1982. p 119).

Muchas de eslas actividades no pasan por el mercado pues se destinan direclamente al consumo familiar. En las sociedades capilalistas, la producción para el inlercambio se vuelve lundamental y la producción para el autoconsumo es secundaria para la circulación del capital. Asi, estas actividades no son consideradas como trabajo y las mujeres que lo realizan no son registradas como Irabajadoras. Es por ello que estos trabajos se calilican como "invisibles".

De esta forma, los economistas se pierden una información valiosa en el estudio de las lormas de reproducción de la fuerza de trabajo en el sislema capitalista y más aún, desconocen la importancia que este trabajo temenino tiene en los tiempos de crisis económica. Algunas respuestas acerca de cómo las lamilias se las ingenian para sobrevivir en tiempos de crisis y de ajuste estructural, o la creciente importancia del sector informal (compuesto en su mayoria por mujeres) vienen de estos

4. Desde el punto de vista de la lógica de la producción campesina, entenderemos trabajo produclivo como aquella actividad que genere ingresos o bienes que serán destinados a la manutención familiar.

5. Sistema agrícola familiar es el conjunto de actividades que realiza la lamilia en torno a la producción agrícola. Incluye actividades tales como: producción agricola directa en el campo, cuido de los animales, limpieza y recolección se semillas, faenas domésticas para mano de obra extrafamiliar, almacenamiento y mercadeo de productos, labores administrativas y toma de decisiones sobre las actividades productivas familiares. 
aspectos olvidados por las clencias Iradicionales.

A manera de ilustración sobre el peso que el trabajo invisible tiene para las famillas e incluso para la economia nacional en su conjunto, se senala que "En términos de valor, las mediciones electuadas sugieren que el trabajo doméstico podría equivaler a un porcentaje que oscila entre un tercio y un medio del ingreso monetario de las tamilias. En el caso de las familias pobres, esa signiticación es mayor, ya que constituye un elemento esencial de la estrategia de sobrevivencia. En Chile se estimo que el aporte del trabajo doméslico de las mujeres al Producto Geográfico Bruto, llegaba al $30 \%$ y sólo era superado por el producto del sector manulacturero" (Irma Arriagada, 1989).

Otros estudios indican que en tiempos de crisis, cuando los empleos escasean y hay dificultades con las tareas agricolas, son las mujeres quienes crean estrategias nuevas para enfrenlarlas. Estrategias que han llevado a algunas a plantearse que son las mujeres con su trabajo las que compensan los electos de la crisis sobre las familias pobres (de Barbieri y de Oliveira, 1989). Más aun, Rosa Paredes consultora de UNIFEM para Centroamérica, ha calificado ese trabajo oculto de las mujeres para la reproducción lamiliar como "ajuste invisible".

\section{Las mujeres salvadorefas en el sistema agricola famlliar.}

En las comunidades visitadas durante el desarrollo del trabajo de graduación, no se encontró una sola mujer que se dedicara exclusivamente al trabajo reproductivo y se pudo constatar que efectivamente colaboran con la búsqueda de ingresos para la manulención familiar. AsI, denlro de la casa misma, la mayoria de las mujeres del lugar se dedicaban a la crianza de aves, venta de huevos, frulos (especialmente aguacales, zunzas y hortalizas cullivadas en forma de huertos caseros), elaboración de dulces, quesos, crema y otros platos tipicos tales como tamales, tortillas, elc; deslinados también para la venla.

Las mujeres además colaboran en la producción de la parcela familiar. Algunas dijeron que durante el inviemo, que es el momento de mayor trabajo agrícola, toda la tamilia se aboca al campo para sacar adelante la pequefía producción. Algunas, las más pudientes, contralan mozos para eslos momentos, la mayoría que no puede hacerlo recurre al uso de la mano de obra lamiliar; "lo que le va a pagar al mozo, mejor que quede para la casa", según dijeron algunas sehoras durante los lalleres realizados. Asi, las nifias y los niños abandonan la escuela y las mujeres las labores domésticas (o mejor dicho, las posponen).

Por otro lado, están también dispuestas a vender su fuerza de traba- 
jo en los momentos en que esto es necesario. De esta forma ellas van a "cortar café durante los meses de noviembre, diciembre y enero de cada ano, trabajan como "mozas" en el caso que la tamilia no posea tierras y otras inclusive van a "rozar" cana en el período de la zafra. En los períodos de verano, que es cuando más se acentúa el desempleo en ol campo, las mujeres tienen mayores dificullades para vender su luerza de trabajo en lo agrícola propiamente y se dedican a otras tareas tales como lavado de ropa ajena, servicio doméstico, elc. Todas estas tareas son más intensas en el caso de las familias más pobres, según se conoció durante el desarrollo de la investigación que este arlículo está siguiendo.

Con todo esto, queda de alguna forma dibujado el hecho que las mujeres si colaboran con la manutención familiar, haciendo muchas veces actividades que se consideran "trabajos de hombre". Sin embargo, siguen siendo las únicas que realizan trabajo doméslico. Independientemente del nivel de pobreza y de trabajo productivo que la mujer realice. el hombre no colabora con el trabajo reproductivo. El resultado es la doble jornada de trabajo para la mujer campesina y mayores dificultades para la realización de trabajo fuera de la casa ya que esla absorbe una buena parte del tiempo y además no pueden simplemente desconectarse de la responsabilidad del cuido de los hijos. Esto como se verá más adelanle, constituye un obstáculo para que la mujer pueda disponer de ingresos o recursos productivos que le ayuden a solventar el problema de la manutención familiar.

Este trabajo incluye las tareas propiamente domésticas de cuido y limpieza del hogar, como otras tales como recoger lena, llevar agua a la casa, elc. Ciertas labores de servicio se cuentan lambién dentro de las responsabilidades de las mujeres, entre estas $s e$ incluye atender con comida y cuido de la ropa al esposo y a los hijos varones. Estas labores de servicio se vuelven obligatorias para las mujeres a lal punto que si no son cumplidas los hombres tienen derecho a enojarse y pueden incluso servir de justificación para algunos aclos de agresión doméstica.

Las mujeres también tienen a su cargo la administración de los recursos que la familia destinará a la satisfacción de las necesidades de sobrevivencia familiar. Esla tarea es derivada del hecho de que ellas son quienes están más pendienles de la lamilia y conocen por lo tanto, los pormenores del manejo de la casa y de las necesidades particulares de los miembros del hogar. Por esa razón las mujeres aportan a la casa todo el dinero que logran ganar, los hombres en cambio se limilan a dar una cuota de su salario para los gastos familiares y ellas deben ingeniarse para que este ingreso alcance para las necesidades de la casa. 
Una de las senoras que asistio a los talleres decla:"..Uno no va a decir que eslá guardando esos centavilos sólo para uno, sino que para la misma casa. Galán fuera que lo que ellos ganan nos lo dieran a nosotras. El sólo da el malz y los trljoles y de alli uno ve que hacer. Por eso hago mis ventecilas para ver de donde saco si hay necesidad..."

Este es un asunto clave en el diseno de políticas y proyectos que intenten proveer una fuente de ingreso a la familia. Los hombres y las mujeres no alrontan con igual responsabilidad las necesidades de la casa, asi mismo no aporlan a ella una lgual proporción sus ingresos. Por tanto, elegir el destinatario directo de alguna actividad generadora de ingresos, no resulta indiferenle a la satisfacción de las necesidades de los miembros de la lamilia.

\section{Mujeres y recursos productivos.}

A pesar que las mujeres trabajan en lo productivo, estas no cuentan con acceso a recursos productivos y sus ingresos son siempre menores que los de los hombres. No es que estos tengan las plenas facilidades para conseguir recursos o ingresos, el campesinado salvadoreno se desenvuelve generalmenle en un mundo bastanle pobre, en cuanlo a las condiciones de vida, los ingresos o salarios que perciben, y con carencias de recursos productivos tales como las tierras, asistencia lécnica y los crédilos. Empero, las mujeres carecen aun más de estos recursos y sus ingresos son todavía más reducidos que los de los hombres campesinos. En el caso del acceso a la tierra, y en el ejemplo especifico de la reforma agraria salvadorena; la $X$ evaluación de la Reforma Agraria presenlada por el PERA en mayo de 1991 sosliene que de 33096 beneficiarios de la lase I de la Relorma sólo 3972, es decir el $11.7 \%$, son mujeres y el resto $(88.3 \%)$ son hombres. Por otro lado, en la lase III el porcentaje de mujeres beneficiarias asciende a solamente un $10.5 \%$ del total, siendo $89.5 \%$ el porcentaje de hombres lavorecidos en esta misma fase.

Duranle el trabajo de campo realizado para el trabajo de graduación se visiló la cooperaliva de la Reforma Agraria "El Sunza de RL", ubicada on el cantón del mismo nombre. Los dalos allí recolectados dan cuenta de que de los 331 asociados con que cuenla la cooperativa a 1991, solamenle 9 son mujeres, es decir el $2.7 \%$ del total. Las mujeres asociadas son vistas con recelo por parte de los socios varones ya que estos han llegado incluso a pedir la expulsión de aquellas durante una asamblea general realizada durante los primeros meses de 1992, según lo manifesló un direclivo de la cooperativa. 
En el resto de los cantones visitados, en los cuales predominan los productores minifundistas, de 33 beneficiarios de la III etapa de la Relorma Agraria solamente 2 eran mujeres, según los registros de la Oticina de Extensión Agropecuaria del Ministerio de Agricultura y Ganaderla, que opera en la zona de Armenia.

La suerte para las mujeres jelas de familia no muestra una gran diferencia con respecto a los patrones generales de discriminación la mujer y si muestra disparidades en cuanto a los recursos que logran poseer los hombres jefes de familia.

Por otro lado, en lo referente al crédito y para el sector no reformado, el Ministerio de Agricullura y Ganaderla da cuenta que para el ano 1988 de un lotal de 27394 crédilos olorgados 5831, o sea el $21 \%$ correspondieron a mujeres y el reslo a hombres. Para 1991-92, la siluación no había variado mucho ya que del lotal de créditos otorgados, apenas el $18 \%$ fue concedido a mujeres.

Con relación a la asistencia lécnica en los cantones visitados se observó que la capacilación impartida por la Oticina de Extensión Agropecuaria del Ministerio de Agricullura y Ganaderla, está dirigida lundamentalmente a los productores y aunque no existe ninguna limitación para que las mujeres acudan a las reuniones, ellas muy pocas veces asislen. La capacilación que si se les proporciona exclusivamente a las senoras es la de los Club de Amas de Casa, impartida por las Educadoras del Hogar. El Ministerio cuenta a 1992 con 70 de estas educadoras para todo el país. Esta capacilación no es necesariamente en cuestiones produclivas ya que la mayorla de las veces tralan sobre asuntos doméslicos, de la salud, etc. Es bastante recienle la idea de incorporar en la asistencia de las educadoras del hogar, técnicas relativas al cultivo y comercialización de huertos caseros, probablemente debido a que las mujeres no son consideradas como productoras agricolas.

El salario y los ingresos son también recursos limitados para las mujeres campesinas. Según las Encuestas de Hogares con Propositos Múltiples, realizadas por MIPLAN en 1985, el ingreso mensual promedio para las mujeres rurales era de 249 colones, mientras que el de los hombres era de 270 . El salario mínimo era también menor para las mujeres ya que éste consistia en 245 colones, Irente al de tos hombres que era de 284 colones. En ambos casos se observa que las mujeres reciben menor dinero por su trabajo que los hombres. En el caso de las actividades que realizan dentro de la casa los ingresos son bastante reducidos y además llegan en forma inconstanle. Para la venta de aves, por ejemplo, deben esperar a que estas crezcan los suliciente y a que lleguen las temporadas en que más se venden y en las que los precios 
suben un poco, como sucede durante la temporada navidena.

Además ellas tiene más dificultades para ser contratadas, son rechazadas de muchos empleos por considerarlas ineficientes, o se les coloca en aclividades consideradas "trabajos de mujeres", que el la mayoría de los casos son peor pagados. $Y$ en algunas otras ocasiones ellas rehúsan trabajar cuando son asediadas sexualmente por sus companeros o jefes.

Además de estas limitaciones para acceder directamente a ciertos recursos, las mujeres tienen que enlrentarse al problema de los compaheros quienes no siempre ponen en común sus recursos y su dinero con toda la familia.

Es importante considerar que en muchas ocasiones se justifica el que no sea la mujer la propielaria de los recursos de la familia, e incluso que su ingreso sea menor que el del hombre ya que liende a pensarse que no es ella la jefa de familia y que es el hombre quien en la mayoria de los casos aporta a la sobrevivencia familiar. Incluso, en políticas dirigidas a los campesinos, tales como la Reforma Agraria, podría pensarse que favoreciendo al jefe de tamilia, se favorece también a ésta. Sin embargo, la realidad parecería indicar algo bastante diferente.

El que los hombres aparezcan como propietarios de la lierra, los insumos, los créditos, la asistencia técnica y el dinero, trae consecuencias negalivas para las mujeres y sus familias, ya que no es seguro que esos recursos y esos dineros sean trasladados del jefe hacia la familia, ya que en varias ocasiones las tierras, el dinero, elc. quedan en los expendios de aguardiente sin llegar hasla el hogar. 0 , como sucede en algunos casos, el hombre abandona la familia llevándose consigo la propiedad de la lierra, la membresía de la cooperativa, elc.

Durante el trabajo de campo, varias senoras que fueron entrevistadas manifestaron que sus esposos gaslaban imporlantes cantidades de dinero en emborracharse y que lo que debería llegar hasta la lamilia se quedaba en el expendio de aguardiente. Por otro lado es trecuente encontrarse con hombres "mujereros" lal y como las senoras de los cantones visitados manifestaron. Estos problemas, contrariamente a lo que suele pensarse, lienen repercusiones importantes en la capacidad de manutención de la familia y dado que no son fenómenos aislados sino más bien muy trecuenles, nos encontramos con que el alcoholismo, la poligamia masculina y el maltralo doméstico, podrian ser fenómenos a considerar en políticas que lengan como mela favorecer a las familias pobres. Los problemas "privados" dejan de ser tales cuando se comprueba que lienen repercusiones sociales serias. 
Por otro lado, el darle la propiedad de la parcela o la membresía de la cooperativa a los hombres, trae problemas a la familia ya que si la unión conyugal se disuelve (como es frecuenle debido a la multitud de casos de abandono e irresponsabilidad palerna), nadie asegura que ese hombre no dejará a su familia en el desamparo. De hecho, cuando esto sucede, lo más frecuente, en los cantones visitados, es que los recursos familiares a disposición del hombre, son llevados con él y puestos al servicio de la nueva Iamilia. No existe en las sociedades cooperativas nl en los beneficiarios del decreto 207 (beneficiarios de la III lase de la Reforma Agraria) alguna obligación para que en estos casos dejen la propiedad o el benelicio a la familia. En el caso de la Cooperativa El Sunza, el gerente dijo que "...eso depende del crilerio de cada asociado..." Si el socio decide disolver la unión conyugal puede, si lo desea, dejar desprovista a su companera y a sus hijos no sólo del sueldo que como cooperativista recibe sino de cierlas lacilidades que como socio también posee. Facilidades tales como el tener prioridad para que sus beneficiarios sean contratados en algunas actividades de la cooperativa, clínica de asistencia, escuela, dividendos al final de la cosecha, etc.

\section{Mujeres y dlscriminación.}

Tal y como se ha visto hasla este momento las mujeres cuemlan con mayores dificullades que los hombres para acceder a recursos productivos y a ingresos que ayuden a la sobrevivencia tamiliar. Las mujeres además cuentan con una sobre carga extra de trabajo que incluye el trabajo por dinero o la generación de productos para la familia, y el Irabajo doméstico/reproductivo destinado al cuido del hogar y de los hijos.

Los limiles que la sociedad le impone a las mujeres para acceder a trabajos mejor remunerados o a la propiedad directa de la tierra, los créditos o acceso a políticas deslinadas al mejoramiento de la vida de las ramilias campesinas, provienen de la posición secundaria que la mujer ocupa dentro de las sociedades patriarcales. Esta posición secundaria tiene consecuencias prácticas y concretas que alectan la vida productiva y reproductiva de las mujeres, y que provocan que se tienda a pensar que las mujeres tienen menor capacidad para manejar los recursos familiares, así como menor eficiencia para ciertos empleos.

El que la mujer sea la responsable única del trabajo doméstico y reproductivo la limita en cuanto al tiempo que estas tareas requieren. No es raro en ese sentido que las mujeres campesinas deban ir a sus trabajos llevando a los nifos consigo, en muchos casos con nifos pequeños. Una de las senoras que se entrevistaron, dijo que ella debia 
llevarse al trabajo en una finca a sus hijos más pequehos y a unos pollos que criaba para vender, y para que la dejaran trabajar sin interrupciones los encerraba juntos, los ninos y los pollos en una champa improvisada por ella misma.

Por olro lado, el que no sean consideradas como las jeias de la Iamilia implica que no son las beneficiarias direclas de algunas políticas que tengan como requisito el ser las jefas. Además el que el jefe de la casa sea el hombre y dadas las atribuciones que a este se le confieren, las mujeres tampoco pueden administrar los recursos con que cuente su familia. Inclusive, tal y como se vio durante el trabajo de campo, hasta para sembrar huertos caseros ellas deben pedirle a "él" que les preste un pedacito de tierra.

En el caso de la Relorma Agraria salvadorefia, el estudio patrocinado por la Fundación Arias (1992) sobre el acceso de la mujer salvadorefia a la lierra sostiene que en la poca participación como beneficiaria directa, la mujer "...se vio alectada (negativamente) por los criterios que establecían que el "jefe de familia" debía ser aceptado como socio y que "la tierra debia ser asignada a quién la trabajan", ya que la participación de la mujer rural en el trabajo es invisible (en el caso del Irabajo doméstico familiar no remunerado y estacional). $Y$ porque la categoria "jele de lamilia" pudo ser interpretada con una carga cultural fuerte que idenlilica como lal al hombre"

También las caracteristicas estruclurales de trabajo de la mujer rural, que incluyen el trabajo reproductivo $y$ el trabajo productivo, se convierlen en un obsláculo por las condiciones en que se realiza, por las habilidades que la mujer desarrolla y la experiencia que acumula...Por otro lado, la casa absorbe buena parte del tiempo y de las energias disponibles de la mujer. En cuanto al trabajo produclivo, la mujer participa en lareas consideradas femeninas y su participación es mayoritaria en el trabajo no remunerado y en el trabajo estacional.

Debido a ello la mujer generalmente no cumple con los requisitos de Irabajador permanente ni de arrendatario ni de aparcero, que la ley establecía para ser considerada como beneliciaria de la Reforma Agraria. Y las trabajadoras permanentes lo eran en tareas propias de mujeres, por lo que su incorporación inicial y a lo largo del proceso se ha limilado a dichas tareas, en algunos casos".

De esta carencia de propiedades se deriva el que las mujeres tampoco son sujetas de crédito, pues no posee garantlas prendarias que son exigidas para la concesión de algunos préstamos. Además como no tienen lierras ni se consideran como productoras agricolas, no son po- 
blación objetivo dentro de la asistencia técnica que se brinda al campesinado.

El no ser las jefes de familia trae además olras dilicultades para las mujeres campesinas ya que detrás de la consideración de una jefalura de hogar masculina se esconde una jererquización sexual que ubica a la mujer en un nivel inferior. Dadas las condiciones de la sociedad machisla, esa consideración implica para ella la ausencia de poder de decisión dentro de la tamilia, la sujeción al esposo de manera análoga a la que presentan los menores de edad (las senoras de Armenia dijeron que deben pedir permiso a sus esposos para salir al pueblo, realizar diligencias fuera del cantón en que se vive, elc). En esle contexlo, fenómenos como la violencia y agresión doméstica aparecen en forma bastanle frecuente.

La jerarquia inlerior de la mujer, la agresión doméslica hacia ella y la limitación en cuanto al acceso y manejo de los recursos tamiliares, son aspectos que deben ser considerados por quienes están trabajando en el otorgamiento de tierras y de créditos. Favorecer a las mujeres con la propiedad de la lierra o de crédilos no es suliciente, ya que ésto de por sl no asegura tampoco que sea electivamente ella quien los maneje y por tanto que la familia se beneficie.

\section{La perspectlva de género y el conoclmlento de la realldad.}

La realidad del campesinado salvadorefio es más compleja de los que usualmente tiende a pensarse ya que además de los problemas derivados de los económico estriclamente, también influyen en la problemática rural, ciertos factores culturales. La perspecliva de género lo que intenta es precisamenle, reconocer las problemáticas derivadas de las diferencias entre los quehaceres, responsabilidades, elc. entre los hombres y las mujeres. Asi mismo orienta los trabajos de promoción o de invesligación a sabiendas de esas formas concrelas (y sublerráneas) de organización social. Prelende además no ignorar los electos diferenciados que una acción política pueda generar para los hombres y las mujeres y los aportes, también diferenciados que los dislintos géneros den a un proceso social particular.

Los problemas derivados del palriarcado que presentan los campesinos tienen dilerenles manifeslaciones lales como: las mujeres no realizan los mismos trabajos que realizan los hombres y no comparten con ellos igual responsabilidades lamiliares. Las mujeres en general se encuentran aladas al trabajo doméstico y limiladas por él para la realización de trabajo productivos en los cuales podrla generar más ingresos 
para la manutención tamiliar. Esto además es preocupamte dado que son las mujeres las que están quedando cada vez más al Iremte de las responsabilidad sobre la sobrevivencia lamiliar, debido a problemas tales como las migraciones, el abandono e Irresponsabilidad paterna y problemas como el alcoholismo y la poligamia masculina.

Las mujeres campesinas están con responsabilidad en la sobrevivencia familiar. Se sabe además que ellas trabajan en labores que producen ingresos o productos que ayudarán a la sobrevivencia tamiliar, que además son quienes están más pendientes y conocedoras de las necesidades de reposición de los miembros de la casa y que ellas además son quienes admistran los recursos que se emplearán directamente en la satislacción de esas necesidades.

Se logró además conocer que a pesar de la responsabilidad que ellas tienen dentro de la casa, no cuentan con los recursos necesarios y no siempre el hecho que el esposo si cuente con esos recursos implica que la familia se beneficiará con ellos.

Los impedimenlos que parecen influir en esa carencia, están relacionados con la jerarquia inferior de la mujer dentro de la familia, su doble jornada de trabajo, la responsabilidad única en el trabajo reproductivo y doméstico y los juicios que sobre la mujer se tienen dentro de la sociedad palriarcal.

Tristemente, los estudiosos se olvidan de indagar sobre la reproducción social del campesinado y dejan de lado importanles fenómenos que explicarian las eslralegias de sobrevivencia familiar y las articulaciones que puedan darse entre la sociedad capitalisla y el mundo campesino. La riqueza de estos aportes ha quedado olvidada y en este momento que los análisis con perspectiva de género se han puesto sobre el tapete, es una buena oportunidad para rescatar melodologias que ayuden a comprender mejor la realidad.

Este entoque ha sido olvidado también por los encargados de las políticas en beneficio de los campesinos. En esios momenlos en que se discule la reconstrucción de las relaciones con el campo y que se intenla realizar una relorma baslante amplia en lo relativo a la tenencia de la lierra, es también un buen momento para reconocer la compleja realidad de los tenedores de tierras. Es momento de plantarse además a quién se quiere realmente beneficiar con las políticas que hoy se desarrollan.

Si es que realmente se quieren modificar las condiciones en que las lamilias rurales se desenvuelven y se quiere además investigar seriamente sobre la siluación del campesinado en general, será pues necesario tomar en cuenta esa problemática particular de género y conside- 
rar tanto los efectos que esta tiene para la reproducción familiar, como el obstáculo o contribución que puedan brindar en algún proyecto o programa que tenga como objetivo mejorar las condiciones de vida de una comunidad.

Si se quieren mejorar las condiciones de vida de las familias campesinas, deberán orientarse acciones que además de corregir sus necesidades maleriales concrelas, reordenen cuestiones como la división genérica del trabajo, discriminación de la mujer y otros derivados del patriarcado que han mostrado ser un IImite al desarrollo comunilario, familiar y personal tanto para hombres como para mujeres.

Finalmente el mejoramiento de las condiciones de vida de las mujeres debe lener en cuenta que hombres y mujeres ocupan un lugar distinto en la sociedad y que sus siluaciones son desiguales entre sl. No puede pensarse en modilicar las situación concreta de la mujer, es decir la pobreza, insalubridad o carencia de algunos servicios; fuera de su contexto de género: división genérica del trabajo, violencia sexista, carencia de poder, instilucionalización de formas de discriminación y responsabilidades domésticas. Las políticas integrales de desarrollo que incluyan una perspecliva de género, los programas y proyectos en los que trabaje en la superación de la división genérica del trabajo, deben articularse para poder olrecer una respuesta concreta a uno de los aspectos olvidados por la planificación para el desarrollo. 